\title{
DETECÇÃO DE PEQUENOS TEORES DE FASE SIGMA E AVALIAÇÃO DOS SEUS EFEITOS NA RESISTÊNCIA À CORROSẪO DO AÇO INOXIDÁVEL DUPLEX UNS S31803
}

\author{
Sérgio Souto Maior Tavares ${ }^{1}$ \\ Jorge Luiz Guerreiro de Castro ${ }^{1}$ \\ Raissa Rodrigues Alves Côrte ${ }^{1}$ \\ Vanessa Moura de Souza ${ }^{1}$ \\ Juan Manuel Pardal ${ }^{1}$
}

\begin{abstract}
Resumo: Os aços inoxidáveis duplex são materiais de alta resistência mecânica e alta resistência à corrosão, fatores que justificam a crescente aplicação destes materiais nos setores químico e petroquímico. As boas propriedades dos aços duplex são atribuídas à microestrutura de proporções praticamente iguais de ferrita e austenita, e às adições de elementos de liga como Cr, Mo e N. Entretanto, os processos de fabricação por conformação a quente e soldagem produzem alterações microestruturais importantes no material, que geralmente conduzem a prejuízos na tenacidade e resistência à corrosão. A formação de fase sigma é uma das transformações microestruturais indesejáveis que podem ocorrer durante a fabricação de equipamentos e componentes mecânicos. Neste trabalho foram investigados os efeitos de pequenas porcentagens de fase sigma na resistência à corrosão do aço inoxidável duplex UNS S31803. São também apresentados resultados sobre a detecção e quantificação de fase sigma por metalografia, medidas de dureza e medidas magnéticas com ferritoscópio.
\end{abstract}

Palavras-chave: aços inoxidáveis duplex, resistência à corrosão, ferritoscópio.

Abstract: Duplex stainless steels are high resistance materials with good corrosion resistance, which explain the large application of this class of materials on petrochemical and chemical sector. The excellent properties of these materials are attributed to the biphasic microstructure with equal parts of ferrite and austenite, and also to the $\mathrm{Cr}$, Mo and $\mathrm{N}$ additions. However, the fabrication process by hot forming and welding produces important microstructural changes in the material, which generally decreases the toughness and corrosion resistance. The sigma phase formation is an undesirable microstructural transformation which happens during the equipments and mechanical components fabrication. At this work the effects of small percentages of sigma phase in the duplex UNS S31803 stainless steel corrosion resistance were investigated. Results concerning sigma phase detection and sigma phase quantification by metallography, hardness and magnetic measurements using the ferritoscope are also presented.

Palavras-chave: duplex stainless steels, corrosion resistance, ferritoscope.

${ }^{1}$ Departamento de Engenharia Mecânica - UFF - ssmtavares@terra.com.br 


\section{INTRODUÇÃO}

O aço inoxidável UNS S31803 é um dos graus comerciais mais populares da classe "duplex", ou seja, com estrutura austenoferrítica. Os principais elementos de liga e respectivos teores médios são: $\mathrm{Cr}-22 \%$, $\mathrm{Ni}-5 \%, \mathrm{Mo}-2,5 \%$ e $\mathrm{N}-0,15 \%$. O índice $\mathrm{PRE}_{\mathrm{N}}$, que se refere à resistência à corrosão por pites e é baseado na composição química da liga [ASM SPECIALITY HANDBOOK, GUNN], é aproximadamente 33. A chamada temperatura crítica de pites (CPT - "critical pitting temperature") em solução $3,3 \% \mathrm{NaCl}$ se situa na faixa de 50 a $55^{\circ} \mathrm{C}$ (GUNN, 2003). Este é um parâmetro que define a temperatura a partir da qual o material vai apresentar formação de pites, podendo ser determinado por ensaios de imersão em solução de $\mathrm{FeCl}_{3}$ (norma ASTM G-48, 2000) ou polarização anódica (norma ASTM G-61, 1986) em diferentes temperaturas.

Operações de soldagem e conformação a quente podem produzir transformações microestruturais indesejáveis nos aços inoxidáveis duplex e superduplex. As presenças de fase sigma $(\sigma)$, fase chi $(\chi)$, ou nitretos de cromo $\left(\mathrm{Cr}_{2} \mathrm{~N}\right)$ em juntas soldadas são responsáveis pelo decréscimo da temperatura crítica de pites (CPT) no metal de solda dos aços duplex e superduplex (LINTON, 2004).

A fase $\sigma$ também fragiliza e endurece $\mathrm{o}$ aço. Trabalhos anteriores, entretanto (GUNN, 2003; NILSSON et al., 2000; CHEN \& WENG, 2002) mostram que o efeito de endurecimento não se revela no ínício da formação de fase $\sigma$, ao passo que a tenacidade é logo afetada por pequenos teores desta fase.

A fase $\sigma$ se forma por resfriamento contínuo e lento a partir de $1000^{\circ} \mathrm{C}$, ou por exposição isotérmica no intervalo de $600^{\circ} \mathrm{C}$ a $1000^{\circ} \mathrm{C}$, sendo mais rápida na faixa de $800^{\circ} \mathrm{C}$ a $900^{\circ} \mathrm{C}$. Via de regra, a fase $\sigma$ surge a partir da ferrita $(\delta)$, onde se concentram os elementos $\mathrm{Cr}$ e Mo que participam e favorecem a formação de $\sigma$. A reação $\delta \rightarrow \sigma+\gamma$ é sugerida por diversos autores (VORONENKO, 1997; JIANG et al., 2003).

Neste trabalho foram produzidas amostras com pequenos teores de fase sigma. Nestas amostras foi realizada a quantificação de fase sigma por metalografia e medidas magnéticas, e a avaliação da resistência à corrosão por ensaios eletroquímicos.

\section{MATERIAIS E MÉTODOS}

Uma chapa de 4,0 $\mathrm{mm}$ de espessura de aço inoxidável duplex com composição química mostrada na tabela I foi cortada em amostras de $30 \times 30 \mathrm{~mm}$ de área para realização dos tratamentos térmicos mostrados na tabela II.

Tabela I: Composição química da liga.

\begin{tabular}{|c|c|c|c|c|c|}
\hline $\mathrm{Cr}$ & $\mathrm{Ni}$ & $\mathrm{Mo}$ & $\mathrm{C}$ & $\mathrm{N}$ & $\mathrm{S}$ \\
\hline 22.3 & 5.44 & 2.44 & 0.02 & 0.16 & 0,001 \\
\hline
\end{tabular}

Tabela II: Tratamentos térmicos realizados.

\begin{tabular}{|l|l|}
\hline Amostra & \multicolumn{1}{|c|}{ Condição de tratamento } \\
\hline 1 & Material como recebido \\
\hline 2 & Isotérmico: $800^{\circ} \mathrm{C} / 5$ minutos \\
\hline 3 & Isotérmico: $800^{\circ} \mathrm{C} / 10$ minutos \\
\hline 4 & Isotérmico: $800^{\circ} \mathrm{C} / 15$ minutos \\
\hline 5 & Isotérmico: $800^{\circ} \mathrm{C} / 20$ minutos \\
\hline 6 & Isotérmico: $800^{\circ} \mathrm{C} / 30$ minutos \\
\hline 7 & $\begin{array}{l}1050^{\circ} \mathrm{C} / 15 \text { minutos seguido de } \\
\text { resfriamento ao forno }\end{array}$ \\
\hline
\end{tabular}

Após os tratamentos térmicos, as amostras foram analisadas com o ferritoscópio da marca Helmut Fischer ${ }^{\circledR}$. Este equipamento mede o teor de ferrita, ou seja, o teor de fase ferromagnética. A medida do ferritoscópio é baseada no método da indução magnética. Um campo 
magnético gerado por uma bobina interage com a(s) fase(s) magnética(s) da amostra. As mudanças no campo magnético induzem uma voltagem proporcional ao conteúdo de fase ferromagnética numa segunda bobina. Essa voltagem é então avaliada. Como a fase $\sigma$ é uma fase paramagnética, gerada a partir da ferrita, sua precipitação deve provocar uma queda no sinal de leitura de fase ferromagnética no ferritoscópio. Em cada amostra foram feitas 8 medições com o ferritoscópio.

As diversas amostras foram também avaliadas por microscopia ótica para quantificação da fase $\sigma . \quad O$ ataque metalográfico utilizado foi eletrolítico em solução $10 \% \mathrm{KOH}$. Este ataque revela a fase $\sigma$ e deixa as fases $\gamma$ e $\delta$ praticamente não atacadas.

A resistência à corrosão por pites de todas as amostras foi avaliada por ensaios de polarização cíclica em solução 3,5\% $\mathrm{NaCl}$. Este ensaio, descrito na norma ASTM G61 [4] foi realizado numa célula de três eletrodos (eletrodo de trabalho, referência e contra-eletrodo de platina). Após o lixamento do eletrodo de trabalho até a lixa 400 e o estabelecimento do potencial de corrosão inicia-se a varredura de potencial no sentido anódico, com uma taxa de $1,0 \mathrm{mV} / \mathrm{s}$, até que se atinja uma densidade de corrente de $1,0 \mathrm{~mA} / \mathrm{cm}^{2}$. Nesse ponto, inicia-se a varredura no sentido inverso, com a mesma taxa até o valor do potencial de corrosão. Cada amostra foi ensaiada em duas temperaturas: $\mathrm{T}_{\mathrm{amb}}$ e $40^{\circ} \mathrm{C}$. O ensaio foi realizado com um potenciostato da marca Omnimetria ${ }^{\circledR}$.

\section{RESULTADOS}

As figuras 1(a-f) apresentam as micrografias das amostras A2, A3, A4, A5, A6 e A7, respectivamente. A tabela III apresenta os resultados de quantificação de fase sigma nas diversas amostras e os valores de dureza obtidos. Conforme esperado, o teor de fase $\sigma$ aumenta com o tempo de exposição a $800^{\circ} \mathrm{C}$. A amostra resfriada ao forno possui teor de $\sigma$ similar ao da amostra tratada isotermicamente a $800^{\circ} \mathrm{C}$ por 10 minutos. Um resultado interessante é que não se observou aumento da dureza devido à fase $\sigma$ nem mesmo na amostra A6 $\left(800^{\circ} \mathrm{C} / 30\right.$ minutos), que apresentou $12,42 \%$ deste constituinte.

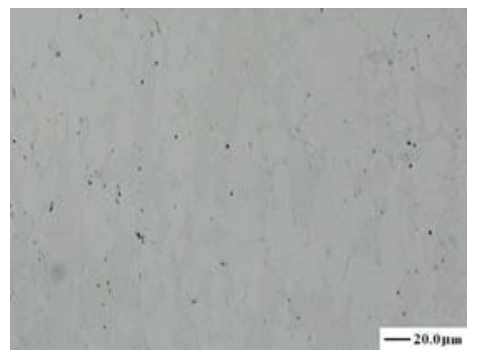

(a)

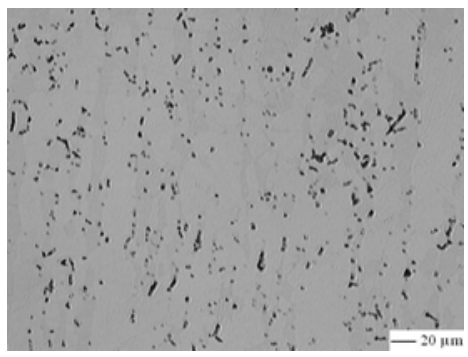

(d)

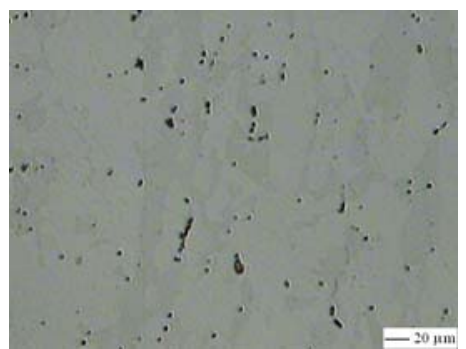

(b)

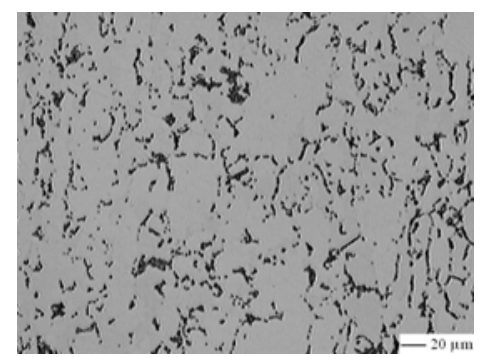

(e)

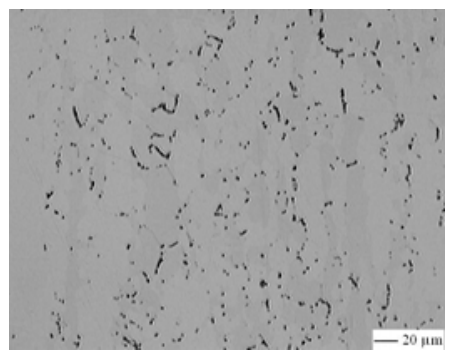

(c)

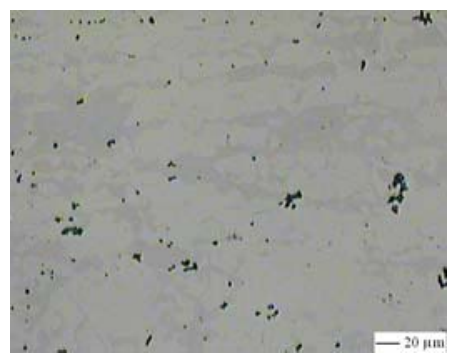

(f)

Figura 1: Micrografias das amostras A2 (a), A3 (b), A4 (c), A5 (d), A6 (e) e A7 (f). 
Tabela III: Percentuais de fase $\sigma$ e valores de dureza nas diversas amostras

\begin{tabular}{|c|c|c|}
\hline Amostra & $\% \sigma$ & Dureza \\
\hline A1 & 0 & $278,5 \pm 3,4$ \\
\hline A2 & $0,52 \pm 0,10$ & $264,7 \pm 1,7$ \\
\hline A3 & $1,70 \pm 0,60$ & $257,6 \pm 0,8$ \\
\hline A4 & $4,01 \pm 0,90$ & $255,9 \pm 2,7$ \\
\hline A5 & $4,47 \pm 1,44$ & $261,6 \pm 1,04$ \\
\hline A6 & $12,42 \pm 1,13$ & $273,7 \pm 3,3$ \\
\hline A7 & $1,71 \pm 0,38$ & $258,0 \pm 3,0$ \\
\hline
\end{tabular}

A figura 2 apresenta as curvas de polarização cíclica a $40^{\circ} \mathrm{C}$ e $\mathrm{T}_{\text {amb. }}$ da amostra na condição solubilizada (A1). Os potenciais de pite, cuja determinação é feita nos pontos indicados por setas nas figuras, são praticamente os mesmos, o que confirma que a temperatura crítica de pites (CPT) nesta amostra é superior a $40^{\circ} \mathrm{C}$. Agora, analisando a figura 3, observa-se que a amostra A2, que apresenta cerca de $0,52 \%$ de fase $\sigma$, possui potencial de pites similar ao da amostra A1 na temperatura ambiente, mas bastante inferior no ensaio realizado a $40^{\circ} \mathrm{C}$. Em outras palavras, pode-se dizer que apenas $0,52 \% \sigma$ é capaz de provocar uma queda no valor da CPT. Os potenciais de pite $\left(\mathrm{E}_{\text {pite }}\right)$ medidos na $\mathrm{T}_{\text {amb. }}$ permanecem elevados nas amostras A3 $(1,70 \pm 0,60 \% \sigma)$, A4 $(4,01 \pm 0,90 \% \sigma)$ e A5 $(4,47 \pm 1,44 \% \sigma)$, mas em todas elas o aumento da temperatura para $40^{\circ} \mathrm{C}$ provoca queda significativa do $\mathrm{E}_{\text {pite. }}$ A amostra A6, que contém cerca de $12,42 \% \sigma$ possui, já na temperatura ambiente, um potencial de pites expressivamente mais baixo do que a amostra A1. A tabela IV apresenta os valores de potencial de pites medidos nas diversas amostras, a $40^{\circ} \mathrm{C}$ e $\mathrm{T}_{\mathrm{amb}}$.

Tão importante quanto quantificar os efeitos de pequenos teores de fase sigma nos aços duplex e superduplex, é detectar esta precipitação de forma não destrutiva. Visto que a formação de fase sigma ENGEVISTA, v. 8, n. 2, p. 77-82, dezembro 2006 provoca uma queda significativa nas propriedades ferromagnéticas do aço, ensaios que avaliem variações na permeabilidade e/ou saturação magnética se constituem em boas opções.

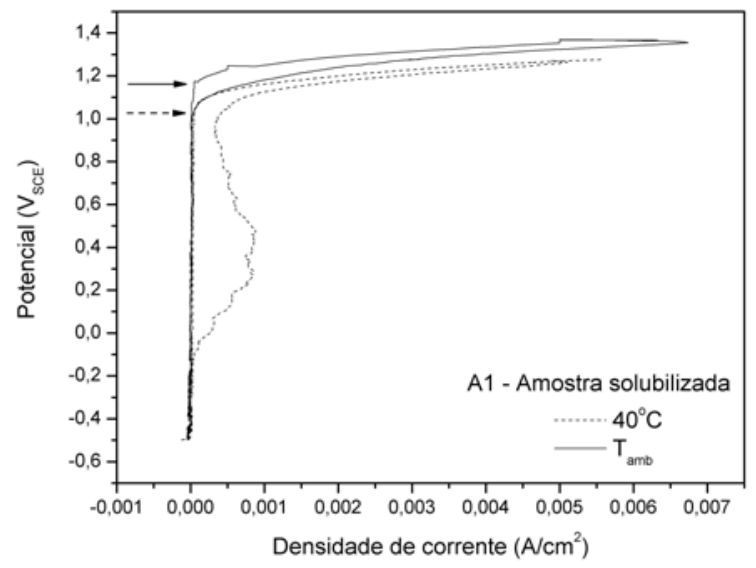

Figura 2: Curvas de polarização da amostra A1.

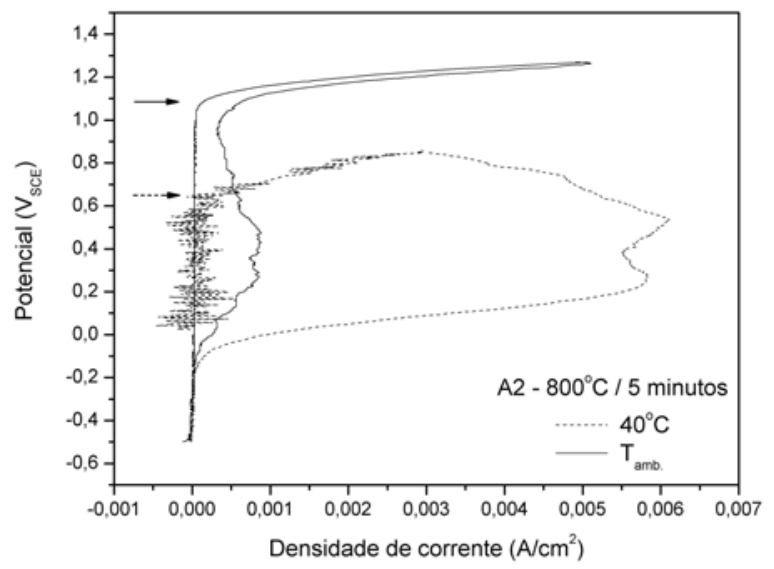

Figura 3: Curvas de polarização da amostra A2.

Tabela IV: Percentuais de fase $\sigma \mathrm{e}$ potenciais de pites medidos.

\begin{tabular}{|c|c|c|c|}
\hline Amostra & $\% \sigma$ & $\begin{array}{c}\mathrm{E}_{\text {pite }} \\
\left(\mathrm{T}_{\text {amb. })}\right.\end{array}$ & $\begin{array}{c}\mathrm{E}_{\text {pite }} \\
\left(40^{\circ} \mathrm{C}\right)\end{array}$ \\
\hline A1 & 0 & 1,17 & 1,07 \\
\hline A2 & $0,52 \pm 0,10$ & 1,15 & 0,65 \\
\hline A3 & $1,70 \pm 0,60$ & 1,16 & 0,54 \\
\hline A4 & $4,01 \pm 0,90$ & 1,11 & 0,69 \\
\hline
\end{tabular}




\begin{tabular}{|c|c|c|c|}
\hline A5 & $4,47 \pm 1,44$ & 1,25 & 0,64 \\
\hline A6 & $12,42 \pm 1,13$ & 0,78 & 0,36 \\
\hline A7 & $1,71 \pm 0,38$ & 1,13 & 0,62 \\
\hline
\end{tabular}

A tabela $\mathrm{V}$ apresenta os valores de $\% \delta$ medidos no ferritoscópio nas diversas amostras. O gráfico da figura 4 mostra o comportamento da $\% \delta$ racionalizado para o valor medido na amostra solubilizada (38,40\%), em função do $\% \sigma$. Neste gráfico são plotados apenas os pontos das amostras tratadas isotermicamente (A1, A2, A3, A4, A5 e A6). Os pontos foram ajustados por uma equação de decaimento exponencial de segunda ordem, dada por:

$$
\begin{aligned}
& \% \delta=0,52 \cdot \exp (-\% \sigma / 22,80)+ \\
& 0,16 \cdot \exp (-\% \sigma / 0,38)+0,32
\end{aligned}
$$

Tabela V: $\% \delta, \% \sigma$ e $\%$ de "outras fases".

\begin{tabular}{|c|c|c|c|}
\hline Amostra & $\% \delta$ & $\% \sigma$ & $\begin{array}{c}\text { outras } \\
\text { fases }\end{array}$ \\
\hline A1 & $38,4 \pm 0,35$ & 0 & 0 \\
\hline A2 & $33,3 \pm 0,54$ & $0,52 \pm 0,10$ & $4,68 \pm 1,00$ \\
\hline A3 & $30,6 \pm 0,69$ & $1,70 \pm 0,60$ & $6,10 \pm 1,64$ \\
\hline A4 & $29,7 \pm 0,91$ & $4,01 \pm 0,90$ & $4,70 \pm 2,16$ \\
\hline A5 & $27,8 \pm 0,53$ & $4,47 \pm 1,44$ & $6,10 \pm 2,32$ \\
\hline A6 & $23,6 \pm 0,30$ & $12,42 \pm 1,13$ & $2,38 \pm 1,78$ \\
\hline A7 & $28,3 \pm 0,50$ & $1,71 \pm 0,38$ & $8,39 \pm 1,23$ \\
\hline
\end{tabular}

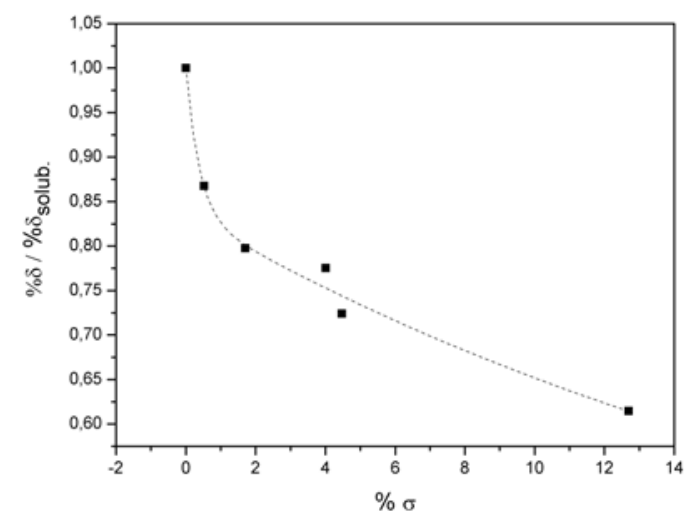

Figura 4: Variação da $\% \delta$ racionalizada para o valor $38,4 \%\left(\% \delta_{\text {solub. }}\right)$ em função da porcentagem de fase $\sigma$.
Voltando aos dados da tabela V, o decréscimo no teor de ferrita não deve ser somente atribuído à precipitação de fase $\sigma$, mas também a outras fases paramagnéticas ou menos ferromagnéticas, tais como nitretos e carbonetos de cromo e, principalmente, austenita secundária. Essas fases também são também consideradas prejudiciais à resistência à corrosão do material (GUNN, 2003; VORONENKO, 1997). A $3^{\mathrm{a}}$ coluna da tabela fornece uma estimativa dos percentuais dessas "outras fases", calculado de acordo com a fórmula:

"outras fases" $=38,4 \%-\% \delta-\% \sigma$

Sendo: $38,40 \%=$ Percentual de ferrita medido pelo ferritoscópio na amostra solubilizada (A1);

$\% \delta=$ Percentual de ferrita delta medido pelo ferritoscópio;

$\% \sigma=$ Percentual de fase $\sigma$ medido por metalografia.

Cabe observar que o valor de ferrita medido no ferritoscópio na amostra A1 $(38,40 \%)$ é inferior ao medido por metalografia $(46,0 \pm 3,6 \%)$. Dessa forma, os resultados mostrados na terceira coluna da tabela $\mathrm{V}$ devem ser encarados de forma qualitativa. Apesar disso, estes resultados ajudam a explicar a diferença de leitura no ferritoscopio entre as amostras A7 e A3 que possuem praticamente o mesmo teor de fase sigma. A amostra A7, por ter sido resfriada lentamente a partir de $1050^{\circ} \mathrm{C}$, apresenta maiores teores de austenita secundária, carbonetos e nitretos, o que explica o seu sinal magnético mais baixo no feritoscópio, apesar de ter o mesmo teor de fase $\sigma$ observado na amostra A3.

$\mathrm{O}$ uso do ferritoscópio como forma de detecção da formação de fase $\sigma$ é, portanto, bastante promissor. São necessários ainda outros ensaios de calibração, para diferentes aços, bem como um aprofundamento maior para os casos em que a fase sigma se forma por resfriamento contínuo. 


\section{CONCLUSÕES}

A formação de apenas $0,52 \%$ de fase sigma por tratamento isotérmico a $800^{\circ} \mathrm{C}$ por 5 minutos foi suficiente para provocar um considerável decréscimo no potencial de pites do aço inox duplex UNS S31803 no ensaio de polarização cíclica em solução $3,5 \% \mathrm{NaCl}$ a $40^{\circ} \mathrm{C}$.

A precipitação de cerca de $12,7 \%$ de fase $\sigma$ provocou o decréscimo do potencial de pites no ensaio de polarização cíclica realizado na temperatura ambiente. A dureza do material, entretanto, é similar à encontrada na condição solubilizada.

A formação de pequenos teores de fase sigma pode ser detectada de forma não destrutiva pelo ferritoscópio. O método é bastante sensível, principalmente nos primeiros estágios de formação da fase $\sigma$.

\section{AGRADECIMENTOS}

Às agências brasileiras de fomento à pesquisa (CNPq, CAPES e FAPERJ) pelo apoio financeiro concedido.

\section{REFERÊNCIAS BIBLIOGRÁFICAS}

ASM Speciality Handbook. Stainless Steels. American Society for Metals, Ohio, 1994.

ASTM G48-00. Standard test methods for pitting and crevice corrosion resistance of stainless steels and related alloys by use of ferric chloride solution, ASTM, West Conshohocken, PA, 2000.

ASTM G61-86. Standart test method for conducting cyclic potentiodynamic polarization measurements for localized corrosion susceptibility of iron-,nickel- or cobalt-based alloys.West Conshohocken, PA: ASTM; 1998.

CHEN, T.H., WENG, K. L., YANG, J. R. The effect of high-temperature exposure on the microstructural stability and toughness property in a 2205 duplex stainless steel,
Materials Science and Eng. V. A338, pp.259-270, 2002.

GUNN, R.N. Duplex Stainless Steelsmicrostructure, properties and applications Abbington Publishing, Cambridge, 2003.

JIANG, Z.I., CHEN, X. Y., HUANG, LIU, X.Y. Grain refinement of $\mathrm{Cr}_{25} \mathrm{Ni}_{5} \mathrm{Mo}_{1,5}$ duplex stainless steel by reat treatment, Heat Treatment, v.A363 p.263-267, 2003.

LINTON, V.M., LAYCOCK, N.J., THOMSEN, S.J., KLUMPERS, A., Failure of a super duplex stainless steel reaction vessel. Engineering Failure Analysis, v.11, p.243-256, 2004.

VORONENKO, B.I. Austenitic-ferritic stainless steels: a state-of-the-art review. Metal Science and Heat Treatment v.39, n.9-10, p.428-437, 1997. 